\title{
Synthesis of palm oil-based fatty methylhydrazide.
}

\begin{abstract}
Fatty methylydrazides (FMHs) have been successfully synthesized from palm oil. Glycerol was produced as a by-product. The synthesis was carried out by reflux palm oil with methylhydrazine in hexane. FMHs have been characterized using elemental analysis, Fourier transform infrared spectroscopy and $1 \mathrm{H}$ nuclear magnetic resonance technique. The results showed that a 6:1 molar ratio of palm oil to methylhydrazine, a round $78 \%$ maximum conversion of palm oil into FMHs and a $10 \mathrm{~h}$ reaction time are the optimum reaction conditions.
\end{abstract}

Keyword: Fatty methylhydrazide; Hexane; Palm oil. 